\title{
A Starphene is Born: Largest and Most Soluble to Date
}
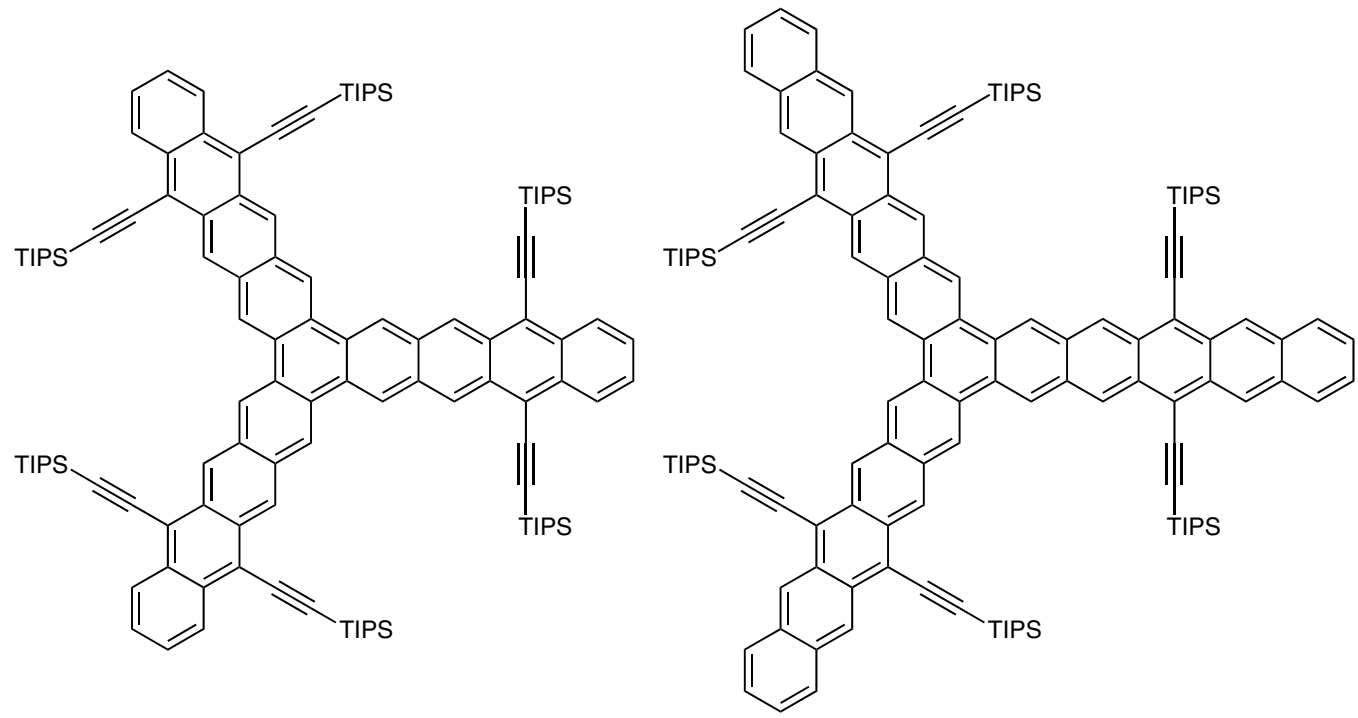

\section{Key words}

electronic materials

starphenes

Yamamoto coupling

Significance: These are the largest reported starphenes to date. Starphenes have garnered attention as organic electronic materials, but previously synthesized versions were extremely poorly soluble and therefore difficult to characterize. The reported structures are more soluble, enabeling NMR and crystallographic data, confirming their structure and packing order into potentially conductive configurations. UV/Vis data indicate that these starphenes have band gaps of 1.8-2.3 eV.
Comment: The starphenes were assembled via a Yamamoto coupling with reasonably good yield ( $\geq 50 \%$ after column chromatography), with crystallographic confirmation of star, rather than linear, structure required. It is also notable that the smaller starphene (left) packed into the predicted 1-D stack, while the larger (right) formed pairs of stacked starphenes that then ordered themselves laterally, which had never before been observed. 